\title{
The Effect of COVID-19 on Routine HIV Care Services from Health Facilities in Northwest Ethiopia
}

\author{
Asmamaw Adugna \\ Jember Azanaw $\mathbb{D}^{2}$ \\ Mequannent Sharew Melaku (D) ${ }^{3}$ \\ 'Department of Health Education and \\ Behavioral Sciences, University of \\ Gondar, Gondar, Ethiopia; ${ }^{2}$ Department \\ of Environmental and Occupational \\ Health and Safety, University of Gondar, \\ Gondar, Ethiopia; ${ }^{3}$ Department of Health \\ Informatics, University of Gondar, \\ Gondar, Ethiopia
}

Background: There is mounting evidence that the risk of death from COVID-19 among people with HIV could be as much as twice that of the general population. Recent evidence revealed that HIV services has been decreased by $75 \%$ and the problem is much more extensive in Ethiopia since most of the logistics for HIV services and fund donated by the good will of NGOs. Understanding the impact of COVID-19 on HIV services is a crucial first step to draw appropriate intervention. Thus, this study aimed to assess the impact of COVID-19 pandemic on HIV services in northwest Ethiopia.

Methods: An institution-based repeated cross-sectional study was conducted in Gondar city in August 2021. The DHIS-2 system, operated by FMOH contains data from all the nine health facilities for HIV care was used to extract data from the central repository. Excel data was exported to STATA 14 for analysis. We calculated indicators of HIV services, representing the 12 months pre-COVID 19 (2019) and 16 months during the COVID-19 period (2020 and 2021). ANOVA was used to detect the presence of significant mean differences between those periods. Assumptions of ANOVA was checked. The statistical significance was declared at $95 \%$ confidence interval $(\mathrm{CI})$, p-value less than 0.05 .

Results: The mean difference was significant within HIV_VCT, HIV_PICT, ART between the years 2019 before COVID-19 and 2020 during COVID-19 (p-value $<0.05$ ). HIV_VCT, ART variability was substantial between the years 2019 and 2021 (p-value $<0.05$ ).

Conclusion: COVID-19 seriously affected all aspects of HIV service uptake such as HIV VCT, HIV PICT, ART, newly started ART, TB screening, and lost to ART follow-up. This study urges optimizing ART delivery mitigation with the ongoing COVID-19 in Ethiopia and beyond, in order to maintain progress toward HIV epidemic control.

Keywords: impact, COVID-19, HIV care services, northwest Ethiopia

\section{Background}

The coronavirus (COVID-19) pandemic is a worldwide public health crisis. ${ }^{1}$ It originally surfaced in the Chinese city of Wuhan on December 31, 2019, and has since spread globally. ${ }^{1}$ The first case (COVID-19) was occurred in Ethiopia on March 13th, 2020 and has had a tremendous impact on all facets of human life since its occurrence. ${ }^{2-4}$ The health-care delivery system accounts for the lion's share of the crisis (COVID-19). ${ }^{5-8}$ Chronic diseases, such as HIV, have been a major cause of morbidity and mortality around the world until recently. Chronic diseases account about sixty percent of all fatalities worldwide. ${ }^{9}$

HIV AIDS is one of the leading causes of death, accounting for around 690,000 deaths each year worldwide. ${ }^{10}$ In 2020, an estimated 37.6 million people globally were infected with HIV, 1.3 million and 160,000 new adults and children was
Correspondence: Mequannent Sharew Melaku

Email mequsharew8@bgmail.com 
infected. ${ }^{11}$ Around $16 \%$ of peoples living with HIV AIDS were unaware of their HIV status, $27 \%$ were unable to get antiretroviral therapy (ART), and $34 \%$ of those on ART were not virally suppressed. ${ }^{10}$ Eastern and Southern Africa are home to $55 \%$ of HIV-positive peoples. Ethiopia is one of the countries in the eastern African region that has very high HIV AIDS burden.

Significant progress has been made in lowering the burden of HIV in order to meet targets sated for reaching very low HIV burden levels by 2030. Even though the Ethiopian government and its allies have made substantial efforts to minimize HIV-related fatalities and illnesses, HIV AIDS remains a severe burden in Ethiopia. According to United Nation AIDS (UNAIDS) data from 2018, roughly $0.9 \%$ of individuals aged 15 to 49 have HIV. ${ }^{12,13}$ HIV positive patients has a risk of twice dying from COVID-19 pandemic compared to the general population. ${ }^{14}$

The UNAIDS observes that the global efforts towards combating HIV/AIDS have been disrupted by the COVID19 pandemic. ${ }^{11}$ COVID-19 poses a severe threat to HIVpositive people and the global response to the virus, including limits on population movement and the suspension of health services due to fears of the virus spreading. ${ }^{12}$ COVID-19 has been claimed to cause up to $75 \%$ of HIV services to be disrupted in several areas. ${ }^{13}$ COVID-19's direct impact on HIV control initiatives could have resulted in serious failures. ${ }^{11,14,15}$ Under plausible worst-case estimates regarding HIV care delays, HIV-related fatalities could rise dramatically during the COVID-19 epidemic. Ethiopia is striving to adapt to the COVID-19 pandemic by focusing on the immediate and ongoing needs of HIV-positive persons. ${ }^{15,16}$

National inventory assessment held in Ethiopia on December 2020, indicated that $82 \%$ of ARVs and all HIV test kits had more than six months stock of supply with no risk category, $14.2 \%$ had four to six months of supply with low-risk category. On September 2020, about 195,237 adult ART clients on the first-line regimens are enrolled on the ART out of the 308,522 adults who are eligible for ART. ${ }^{11}$

During the lockdown period in $\mathrm{Zambia}^{15}$ there was a $35 \%$ decline in the number of HIV tests conducted, similarly the lockdown period in Ethiopia significantly disrupted the HIV service. Since the focus of Ethiopia is combating COVID-19 infection from the moment the first local cases were detected. As COVID-19 prevention measures became more stringent, hospital visits were discouraged, people were advised to stay at home, and (public) gatherings were restricted in order to prevent the virus from spreading. Furthermore, disruptions in key HIV commodities' supply chains, logistics, and reporting systems have hampered the ability to maintain HIV-related services. In another area, HIV programs that received direct support had their budget curtailed as governments retreated to focus on their COVID-19 load. ${ }^{17}$ This has had a direct impact on the level at which HIV programs are implemented. ${ }^{18}$

It is strongly suggested that the COVID-19 response could be guided by and founded on community-centered and rights-based initiatives. ${ }^{18}$ Understanding the impact of COVID-19 on HIV services is a critical first step in determining the best course of action. As a result, the aim of this study was to see how COVID-19 affects HIV care.

\section{Methods}

\section{Study Design and Setting}

An institution-based repeated cross-sectional/interrupted time series study was conducted in Gondar city in August 2021. Gondar city is located at about $727 \mathrm{~km}$ away from Addis Ababa, the capital city of Ethiopia, $180 \mathrm{~km}$ away from Bahir Dar, the capital city of Amhara Regional State. The city has a total population of 338, 646 with 256,041 whose age is between 18 and 65 years, in 6 sub-cities and 27 kebeles with a total of 78,772 households. There are 9 public health institutions delivering HIV care ( 8 health centers and 1 specialized hospital) and more than 40 private health facilities in the city administration. About 51,990 clients are following the HIV services in all the 9 health institutions.

\section{Study Participants}

All clients who were on HIV care services follow-up in Gondar city public health facilities were during the study period were included in this study.

\section{Study Period}

The study period was from March 2019 to June 2021. During this period there was Pre Covid period which was a period before March 2020; Lock down period, which is part of Covid period, was a period from April 2020 to July 2020; Covid period was a period from March 2020 to up-to-date.

\section{Data Source}

The DHIS-2 system is operated by federal ministry of health (FMOH). The district health information system 2 (DHIS-2) central data repository, located in northwest Ethiopia, contains data from all the 9 health facilities for HIV clinical care management. Data were extracted from 
the central repository in August 2021. All health facilities were replicating their data to the central repository; this ensured that the central data mirrored the data available at the sites through the full data recording period. Excel data were transformed to Stata 14 for analysis.

\section{Outcomes of Interest}

The study included patient data aggregated in monthly values at the health facility level. Our study sought to assess time trends before and after the confirmation of the first cases of COVID-19 in Ethiopia on March 19, 2020, used as a starting mark of "COVID-19 period".

HIV_VCT is human immune deficiency virus voluntary counseling and testing services which is detecting HIV serostatus voluntarily to identify those who need ART care.

HIV_PICT is human immune deficiency virus provider-initiated counseling and testing services, which is detecting HIV serostatus by health-care providers' recommendation to identify those who need ART care.

ART is antiretroviral therapy which is the treatment for the human immunodeficiency virus (HIV) infection using a combination of antiretroviral drugs.

\section{Data Management and Analysis}

Data cleaning, recoding, transforming using Excel version 2016 and STATA version 16.0 softwares. For the missing data, imputation technique was done by filling in to create a complete data matrix so as to analyze using standard method without defining an explicit model for it. Descriptive statistics, counts, means, and tables and graphs were used to describe the characteristics of the study participants and present the study results. We calculated indicators of HIV services in August 2021, representing the 12 months pre-COVID (2019) and 16 months during the COVID-19 period (2020 and 2021) data with the $95 \%$ confidence interval (CI) across the 9 health facilities over the 28 months of the study. Time series analysis was done. Analysis of variance (ANOVA) was used to detect the presence of significant mean differences for HIV care services indicators prior to COVID-19 occurrence and during COVID period. All assumptions of ANOVA were checked. The statistical significance was declared using a p-value less than 0.05 .

\section{Ethical Consideration}

The research proposal was approved and ethical clearance reference number $\mathrm{V} / \mathrm{P} / \mathrm{RCS} / 05 / 574 / 2020$ was obtained from the institutional review board (IRB) of the University of Gondar. Moreover, support letters and permission were obtained from Gondar city health office. However, the study used only aggregated, de-identified data, and no investigators interacted with human subjects or had access to identifiable data or specimens for research purposes, so a consent waiver was obtained for the study. Furthermore, the data were analysed anonymously.

\section{Results HIV_VCT and HIV_PICT at Health Facilities in Northwest Ethiopia}

A total of 51,990 HIV_VCT participants from nine health facilities were reported within 28 months. Only few $(0.998 \%)$ proportions of the participants were children and the most $(88.165 \%)$ them were adults (25-49 years) year old. The overall decreased in HIV_PICT was greater proportion (64.373\%) among male adults (20-59 years) (Table 1$)$.

\section{Trend Analysis of HIV_VCT, HIV_PICT and ART Attendants}

In response to an intense increase in notified COVID-19 cases, especially in Ethiopia from March 2020, and the government again ordered a full national lockdown to the next months. The mean difference was significant within HIV_VCT, HIV_PITC, ART between the years 2019 before COVID and 2020 during COVID (p-value $<0.05$ ). As well as HIV_VCT, ART variability was substantial between the years 2019 and 2021 (p-value < 0.05). But the mean difference of HIV_VCT was not significant within 2020 and 2021 (Table 2). there was a large decline in Voluntary Counselling and Testing (VCT), Provider Initiated Testing and Counseling (PITC) attendances from March 2020 to June 2020. ART delivery was fundamentally continued during lock down although HIV_VCT testing and HIV_PICT were more severely reduced (Figure 1).

\section{Number of HIV_VCT and HIV_PICT Attendants}

Early data before starting COVID-19 showed that the numbers of HIV_VCT and HIV_PICT records was higher. Even there is highly variabilities, between March 2019 before COVID-19, to May, in 2020 during COVID-19, people attending for HIV_VCT was reduced from 4941 to 568. However, there was a total increment of HIV_VCT from October 2020 to the next months (Figure 2). 
Table I HIV_VCT, HIV_PICT and ART Distribution with Sex and Age at Health Facilities in Gondar City

\begin{tabular}{|l|l|l|l|l|l|l|}
\hline \multicolumn{2}{|l|}{} & \multicolumn{2}{l|}{ HIV_VCT } & HIV_PICT & \multicolumn{2}{l|}{ ART } \\
\hline Sex, Age & Frequency (n) & Percent (\%) & Frequency (n) & Percent (\%) & Frequency (n) & Percent (\%) \\
\hline Female, Children (0-14) years & 234 & 0.449 & 3199 & 3.702 & 17 & 1.709 \\
\hline Male, Children (0-14) years & 286 & 0.549 & 3070 & 3.552 & 25 & 2.513 \\
\hline Female, Adolescence (15-19) years & 1269 & 2.441 & 1915 & 2.216 & 33 & 3.317 \\
\hline Male, Adolescence (15-19) years & 4364 & 8.394 & 6766 & 7.828 & 22 & 2.211 \\
\hline Female, Adult (20-59) years & 24,977 & 48.042 & 15,844 & 18.33 & 546 & 55.076 \\
\hline Male, Adult (20-59) years & 20,860 & 40.123 & 55,641 & 64.373 & 350 & 35.176 \\
\hline
\end{tabular}

\section{Newly ART Starting and Lost PLHIV During COVID - 19}

The outcomes of the number of newly ART starting initiations per month, and PLWHA lost visits per month at participating health centers during the study period. The number of monthly ART visits was a function of when visits were scheduled, and whether patients attended these scheduled visits. Since visit planning might also have changed during lockdown, the assessed a secondary consequence of missed ART collection visits per month. Before COVID-19, number of newly started ART at May 2019 was 49 but during lockdown it was reduced to 15 . The other temporary decline of ART was at January 2021, Christmas time that later recovered. PLWHA visit was highly (107) missed during Ethiopian New Year September 2019 before COVID-19 but it increased when COVID-19 started March 2020 and during lockdown (Figure 3).

\section{Number of Clients Enrolled in TB Screening Among HIV_VTC Participants} The other health service particularly disrupted by COVID-19 was TB. The continuing numbers presenting with TB screening the pre-COVID-19, and during COVID-19 lockdown periods are revealed in Figure 4. Compared TB diagnosis and screening services was decreased from April 2019 (8402) before COVID-19 to the lowest record November 2020 (1438) during lockdown in the health-care facilities. However, the trend of TB diagnosis and screening services graph seems increased since January 2021 (Figure 4).

\section{Discussion}

Since December 2019, the occurrence of COVID-19 has had a tremendous impact on all facets of human life around the world. The objective of this study was to see how the COVID-19 pandemic affected HIV services in Ethiopia. This study assessed six components of HIV care services: HIV VCT, HIV PICT, ART, newly started ART, TB screening, and lost to ART follow-up. It is undeniable that the COVID-19 infection has had a substantial impact on the HIV care unit's services.

From April to September 2020, the number of people attending HIV testing decreased dramatically. After September 2020, there was a significant increment in HIV testing. There was a significant mean difference HIV_VCT between the years 2019 (pre-COVID 19) and 2020. Moreover, there was a significant mean difference of HIV_VCT between the years 2019 and 2021. As a result, COVID-19 infection has had a substantial impact on HIV VCT services. The findings of other studies also indicated that HIV testing services were reduced during the first season of the COVID-19 pandemic. ${ }^{10,19-21}$ This could be because governmentmandated lockdowns, which are intended to prevent the spread of COVID-19, have had a wide-range impact on health-care delivery. Furthermore, there was widespread public concern about contracting COVID-19 and being diagnosed with the condition, which resulted in a significant drop in VCT. ${ }^{22}$ COVID-19 also caused a decrement of HIV _ VCT service since health institution was considered as unsafe for visit. COVID-19 poses a greater danger to people who reside in lowand middle-income countries, as well as those who live in impoverished areas. This means that after the first and second waves of COVID-19, African countries should solidify their health systems and handle potential third waves to increase the number of people who enrolled in HIV_VCT care service. HIV_VCT service 
Table 2 Mean and Mean Difference of HIV_VCT, HIV_PICT and ART Attendants

\begin{tabular}{|c|c|c|c|c|c|c|}
\hline \multicolumn{7}{|l|}{ Variables } \\
\hline \multicolumn{3}{|l|}{ Year } & \multicolumn{2}{|c|}{$\begin{array}{l}\text { No. of Persons on } \\
\text { HIV_VCT }\end{array}$} & $\begin{array}{l}\text { No. of } \\
\text { Persons on } \\
\text { HIV_PITC }\end{array}$ & No. of Persons on ART \\
\hline \multirow{3}{*}{\multicolumn{2}{|c|}{2019 (before COVID) }} & Mean & \multicolumn{2}{|c|}{$2,271.58$} & $3,492.25$ & 36.83 \\
\hline & & $N$ & \multicolumn{2}{|c|}{27,259} & 41,907 & 119,940 \\
\hline & & Std. D & \multicolumn{2}{|c|}{281.307} & 696.626 & 7.998 \\
\hline \multirow{3}{*}{\multicolumn{2}{|c|}{2020 (during COVID including lock down) }} & Mean & \multicolumn{2}{|c|}{$\mathrm{I}, 4 \mathrm{I} \mid \mathrm{I} .30$} & $2,373.50$ & 29.40 \\
\hline & & $N$ & \multicolumn{2}{|c|}{14,113} & 23,735 & 11,195 \\
\hline & & Std. D & \multicolumn{2}{|c|}{654.981} & $1,011.196$ & 10.233 \\
\hline \multirow[t]{3}{*}{2021 (post lockdown) } & \multicolumn{2}{|c|}{ Mean } & \multicolumn{2}{|c|}{$\mathrm{I}, 769.67$} & $3,465.50$ & 36.00 \\
\hline & \multicolumn{2}{|l|}{$\mathrm{N}$} & \multicolumn{2}{|c|}{10,618} & 20,793 & 64,547 \\
\hline & Std & & 142 & & 188.205 & 86.251 \\
\hline Mean Difference in Multiple Comparison & by Years & & & & & \\
\hline Dependent Variable & (I) Year & & lear & Mean Difference (I-J) & Sig. & $95 \% \mathrm{Cl}$ \\
\hline & & & & & & Lower Bound-Upper Bound \\
\hline HIV_VCT & 2019 & 20 & & $630.950 *$ & 0.038 & $34.440-1227.460$ \\
\hline & & 20 & & $455.033^{*}$ & 0.002 & $|78.390-73| .670$ \\
\hline & 2020 & 20 & & $-630.950 *$ & 0.038 & $-1227.460-(-34.440)$ \\
\hline & & 20 & & -175.917 & 0.708 & $-757.120-405.290$ \\
\hline & 2021 & 20 & & $-455.033 *$ & 0.002 & $-731.670-(-178.390)$ \\
\hline & & 20 & & 175.917 & 0.708 & $-405.290-757.120$ \\
\hline HIV_PITC & 2019 & 20 & & $1029.000 *$ & 0.029 & $95.130-1962.870$ \\
\hline & & 20 & & 79.500 & 0.946 & $-598.270-757.270$ \\
\hline & 2020 & 20 & & $-1029.000 *$ & 0.029 & $-1962.870-(-95.130)$ \\
\hline & & 20 & & $-949.500 *$ & 0.017 & $-1724.590-(-174.410)$ \\
\hline & 2021 & 20 & & -79.500 & 0.946 & $-757.270-598.270$ \\
\hline & & 20 & & $949.500 *$ & 0.017 & $|74.4| 0-1724.590$ \\
\hline No. of Adults and Children currently on ART & 2019 & 20 & & $-1454.433 *$ & 0.001 & $-2062.190-(-846.670)$ \\
\hline & & 20 & & -762.833 & 0.010 & $-1878.420-(-267.110)$ \\
\hline & 2020 & 20 & & $1454.433 *$ & 0.001 & $846.670-2062.19$ \\
\hline & & 20 & & 381.667 & 0.267 & $-313.22-1076.560$ \\
\hline & 2021 & 20 & & 762.833 & 0.084 & $-93.360-1619.020$ \\
\hline & & 20 & & -381.667 & 0.267 & $-1076.560-313.220$ \\
\hline
\end{tabular}

Note: *The mean difference is significant at the 0.05 level. 


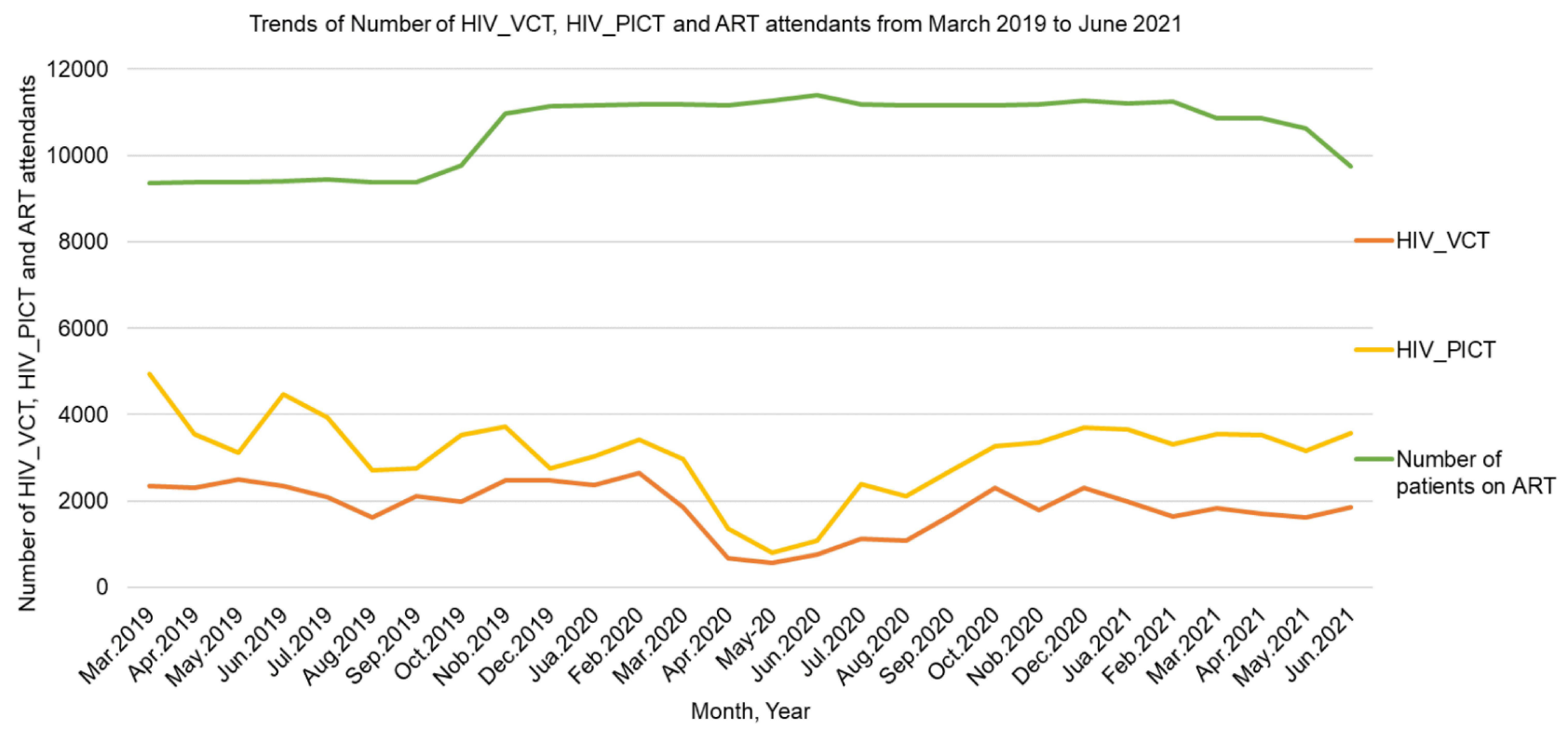

Figure I Number of HIV_VCT, HIV_PICT and ART attendants against Time in Months. In the figure the green colored line represents number of ART attendants, the yellow-colored line represents HIV_PICT and the bright Orange colored line represents HIV_VCT.

6000

Number of HIV_VCT and HIV_PICT attendants from March 2019 to June 2021

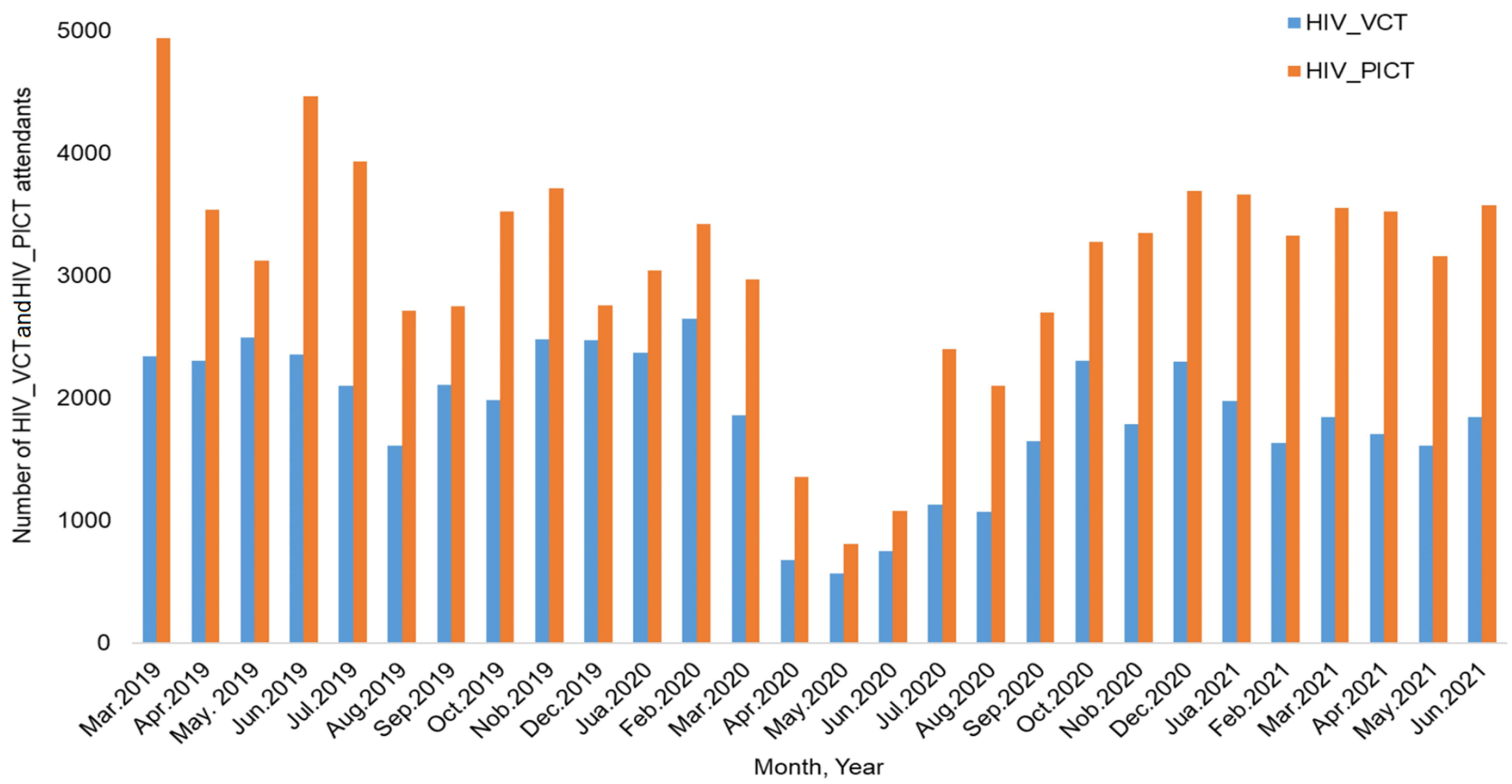

Figure 2 Number of HIV_VCT and HIV_PICT Attendants within Twenty-eight months. In the figure the blue colored bar represents HIV_VCT and the bright Orange colored bar represents HIV_PICT.

is a basic tool to satisfy clients need for being diagnosed for HIV AIDS Accordingly, policymakers could be guided on different scientific papers in order to improve their approach for successful strategical implementations for the sake of increasing people who involved in HIV_VCT service. 


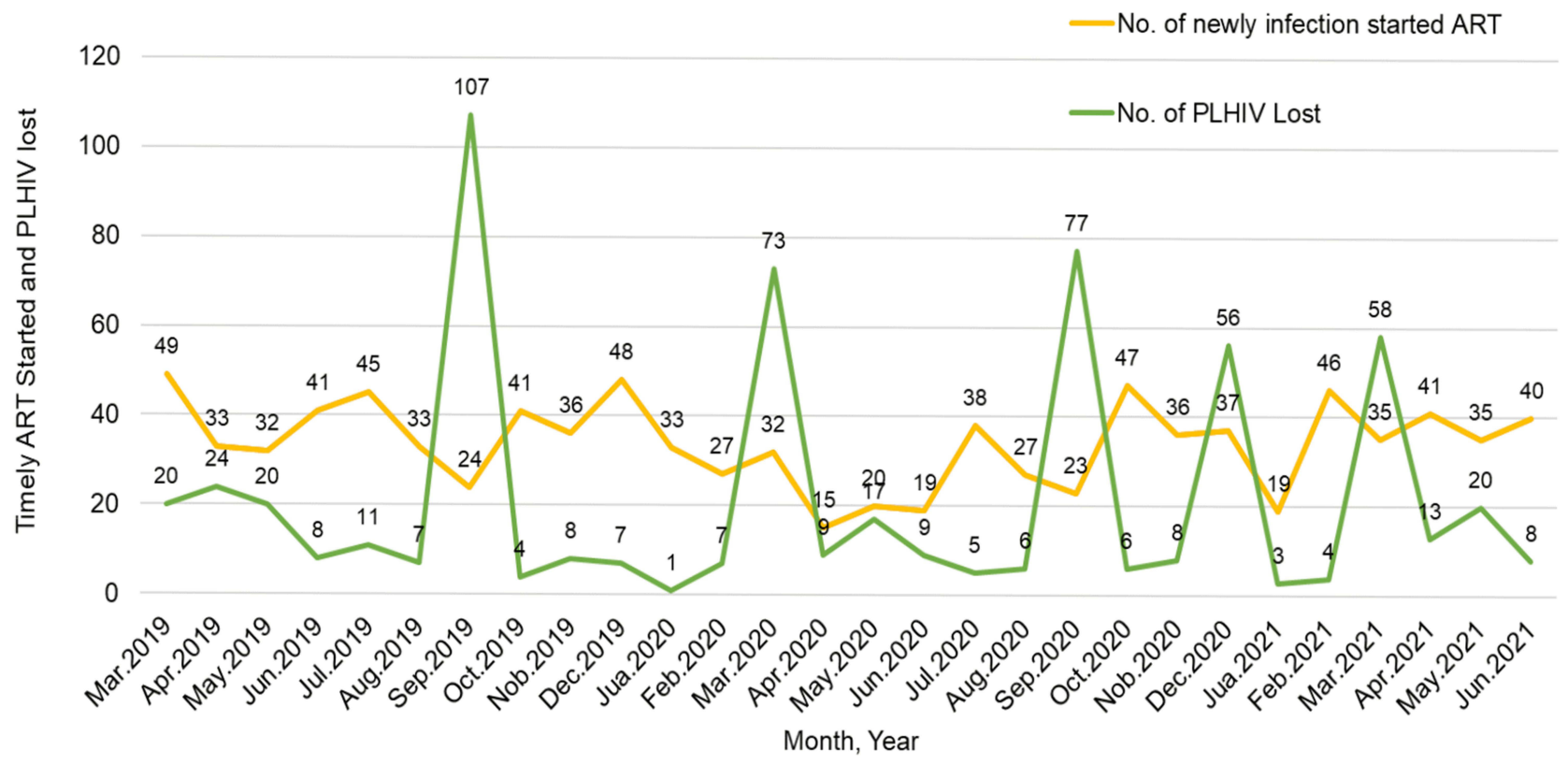

Figure 3 Newly initiation of ART and lost PLWHA of participants' pre COVID-19, during and post COVID-19 lockdown periods in 28 Months. In the figure the green colored line represents number of peoples lost HIV follow up, the yellow-colored line represents number of individuals started ART.

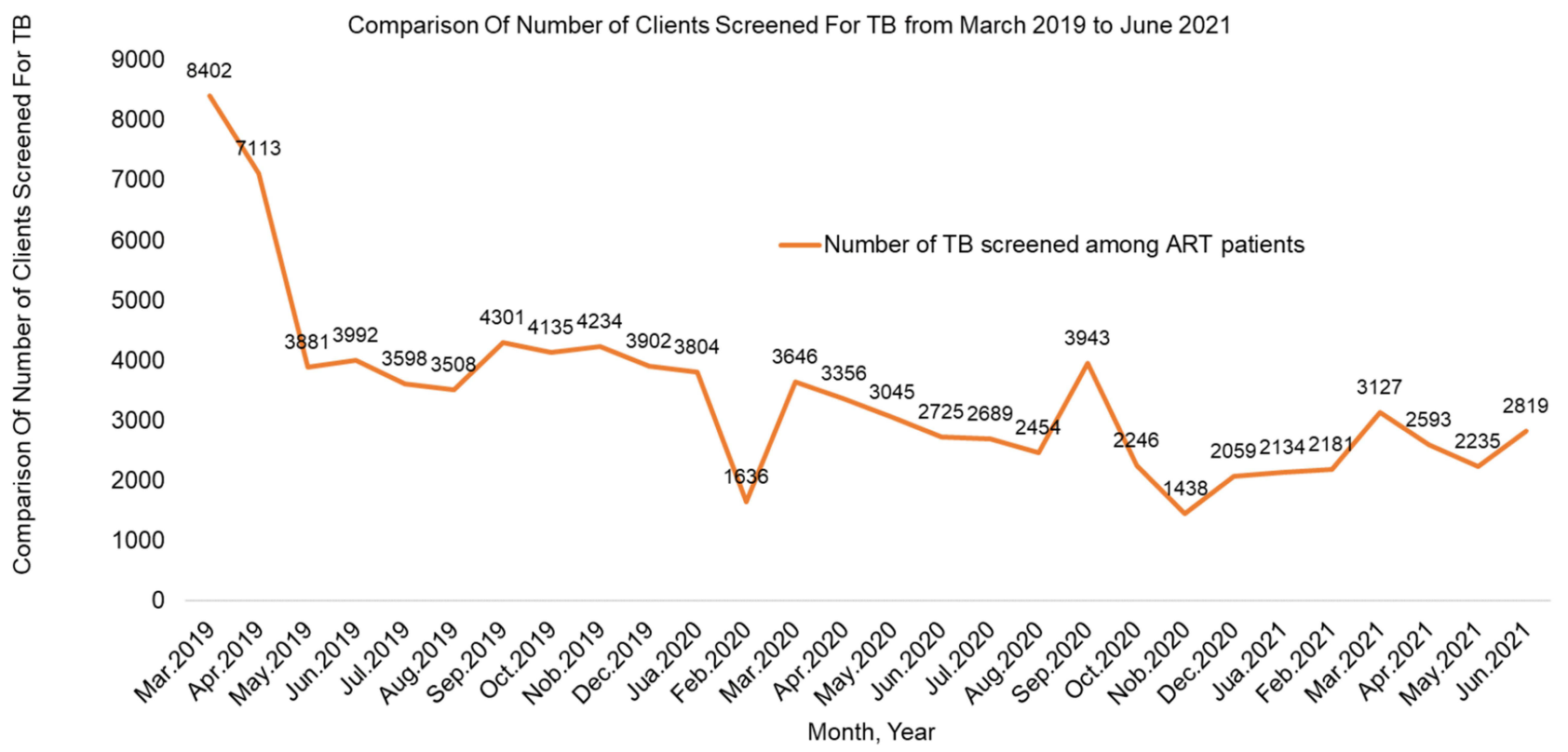

Figure 4 Comparison of number of clients screened for TB of participants' before COVID-19, during and post lockdown periods in 28 Months. In the figure the bright Orange colored line represents number of ART patients screened for tuberculosis.

During the first season of the COVID-19 epidemic, the number of patients starting HIV PICT testing was dropped sharply. After September 2020, there was a significant increase in HIV PICT testing initiated by providers. Between the years 2019 and 2020, there was also a statistically significant mean difference in HIV PICT service. The mean difference of HIV PICT also sustained between the years 2019 and 2021. different findings also showed that HIV PICT service was similarly lowered during the first season of the COVID-19 epidemic. ${ }^{19,23}$ This could be because government-mandated lockdowns, which are intended to prevent the spread of COVID-19, 
have had a wide-ranging impact on health-care delivery. As a result, COVID-19 infection has had a substantial impact on HIV PICT services. The health sector received governmental attention, health-care staff, resources, and funds at the start of the COVID-19 pandemic to help it cope with the approaching disaster. There was also a quarantine, restricted movement, and an increase in the amount of time people spent indoors. All of these raised fears that countries with high rates of human immunodeficiency virus/acquired immune deficiency syndrome (HIV/AIDS) would be unable to offer their patients with consistent and high-quality healthcare. Accordingly this all problems affected patients' health-seeking behavior and access to care would be harmed as well. ${ }^{24}$ HIV PICT services is very interesting strategies in order to hunt peoples who are HIV positive when they are going to diagnosed for different chronic health problems. Accordingly, policymakers and organizations who are working on HIV prevention and treatment could focused on different strategical approaches that could better hunt those individuals so as to improve HIV care services.

Adults seeking ART services were also shown to be persistent before and during covid times, including lockdown from April to September 2020, according to the study. After September 2020, there was a significant drop in the number of adults seeking ART. However, there was a considerable mean difference in ART service between 2019 and 2020. These findings indicate that HIV services were generally maintained for patients who were already on antiretroviral therapy (ART). Other earlier investigations backed up this conclusion. ${ }^{25-31}$ The reason for this could be because patients who are already on ART treatment require close monitoring in order to protect them from opportunistic infection and COVID-19 infection through the use of preventive measures. As a result, patients on antiretroviral therapy (ART) may exhibit a similar trend during the pre-COVID and COVID pandemic periods. This implies that the ART service could be continued in the pattern that exhibits in this finding. However, the quality of life among patients who are receiving ART is still doubtful. Accordingly, policymakers could invest their maximum efforts to improve the quality of life among patients who are involved in HIV care.

The other major health service affected by COVID-19 was TB diagnosis and screening services among people living with HIV AIDS. TB diagnosis and screening services in health-care facilities fell from April 2019 (8402) before lockdown to the lost record November 2020 (1438) during lockdown. This finding is supported by a variety of findings. This could be due to COVID-19 lockdowns, which are necessary to prevent the virus from spreading. Furthermore, TB screening necessitates some form of contact between physicians and ART patients, which could enhance the transfer of COVID-19 infection from physicians to patients and vice versa, given that more than half of COVID-19 infections are asymptomatic, according to various studies. As a result, patients who are on ART may receive less attention from TB screening programs. The primary aim of improving quality of life among ART patients is screening and treating opportunistic infections. Tuberculosis infection is among the commonest opportunistic infection that frequently affects the quality of life among ART patients. Thus, policymakers could design different strategies that could enhance tuberculosis screening during the persisting COVID-19 period in the study setting in particular and in Ethiopia in general.

The number of ARTs that have recently begun was lowest ${ }^{15}$ newly starting ART was observed following shutdown in May 2019. Another small drop in ART occurred in January 2021, at Christmas time, which was quickly restored. In addition to COVID-19, PLWHA visits were heavily (107) missed during Ethiopian new year in September 2019, but have since been decreased to eight since July 2021. Patients who were already receiving HIV treatment and were aware of the need of maintaining high adherence may have prioritized ART collection. People were stockpiling up in anticipation of anticipated interruptions, according to ART collection trips. Clinics were also able to make ART more accessible by implementing tactics like multi-month prescribing and customized service delivery. The primary aim of hunting peoples with HIV AIDS through HIV_VCT and HIV_PICT is to ensure that, patients are being involved in HIV care. Accordingly, assessing patients in pre-ART service and initiating ART treatment is the first steps of HIV care and all patients diagnosed as HIV positive could be started ART treatment. Thus, policymakers could work on initiation of ART treatment for all patients diagnosed HIV positive.

Similarly, the number of patients who were lost to follow-up for ART treatment was 107 in the pre-OCVID infection period, there after it steadily declined to 73 at the start of COVID-19 infection, and then increased slightly to 77 in September 2020. This could be because most patients who have recently started ART treatment have experienced a lost to follow-up. COVID-19 infection, on 
the other hand, reduced HIV VCT and HIV PICT, according to this study. This, in turn, correlates to a decrease in new ART initiation and missed ART treatment appointments. The primary focus of HIV care is not only initiating ART treatment but also sustaining patients throughout their life span. Accordingly, policymakers could design practicable and community-based approaches to hunt patients who lost their ART follow-up.

\section{Strength and Limitation}

This is the first paper to examine the impact of COVID-19 on HIV care services in Ethiopia. As to limitation this paper only examines the trends of COVID 19 impacts on HIV AIDS. Thus, it lacks modeling determinant factors of COVID 19 impacts on HIV AIDS.

\section{Conclusions}

COVID-19 seriously affected all aspects of HIV service uptake such as HIV VCT, HIV PICT, ART, newly started ART, TB screening, and lost to ART follow-up. The sudden changes in HIV service utilization represent dramatic adaptations needed to mitigate primary and secondary effects of the COVID-19 pandemic on PLWHA.

\section{Recommendations}

This study urges optimizing ART delivery mitigation with the ongoing COVID-19 in Ethiopia and beyond, in order to maintain progress toward HIV epidemic control. In addition, a solid community-based strategies could be designed in order to improve the care for HIV patients during this critical COVID-19 period. Community mobilization and community-based HIV testing and counselling could be encouraged.

\section{Abbreviations}

AIDS, acquired immune deficiency syndrome; ANOVA, analysis of variance; COVID-19, coronavirus disease 2019; HIV, human immune deficiency virus; HIV AIDS, human immune deficiency virus acquired immune deficiency syndrome; NGO, non-governmental institution; CI, confidence interval; DHIS2, District Health Information System 2; FMOH, Federal Ministry of Health; HIV_PICT, human immune deficiency virus provider initiated counselling and testing; HIV_VCT, human immune deficiency voluntary cancelling and testing; ART, antiretroviral therapy; PLWHA, people living with HIV AIDS; IRB, Institutional Review Board; TB, tuberculosis; STATA, Stat Corp software; UNAIDS, United Nation AIDS.

\section{Data Sharing Statement}

All relevant data are in the manuscript. However, the minimal data underlying all the findings in the manuscript will be available upon request.

\section{Acknowledgments}

We are grateful to the staffs of Gondar city health facilities for their cooperation for the feasibility of this study by providing all the necessary data to enable us to conduct the study.

\section{Author Contributions}

All authors made a significant contribution to the work reported, whether that is in the conception, study design, execution, acquisition of data, analysis and interpretation, or in all these areas; took part in drafting, revising or critically reviewing the article; gave final approval of the version to be published; have agreed on the journal to which the article has been submitted; and agree to be accountable for all aspects of the work.

\section{Funding}

No funding was obtained for this study.

\section{Disclosure}

The authors report no conflicts of interest in this work.

\section{References}

1. World Health Organization. Coronavirus disease 2019 (COVID-19): situation report, $94 ; 2020$

2. Oosthuizen M, Cilliers J, Pooe T, Alexander K, Moyer JD. Impact of COVID-19 in Africa: a scenario analysis to 2030. ISS Afr Rep. 2020;2020(24):1-40.

3. Taffesse A, Minten B. Response to the Covid-19 pandemic in Ethiopia-some reflections. Research Note Washington DC: IFPRI; 2020.

4. Wondimu W, Girma B. Challenges and silver linings of COVID-19 in Ethiopia-short review. $J$ Multidiscip Healthc. 2020;13:917. doi:10.2147/JMDH.S269359

5. Pillay Y, Pienaar S, Barron P, Zondi T. Impact of COVID-19 on routine primary healthcare services in South Africa. $S$ Afr Med J. 2021;111(8):714-719. doi:10.7196/SAMJ.2021.v111i8.15786

6. Eisinger RW, Lerner AM, Fauci AS. Human immunodeficiency virus/ AIDS in the era of coronavirus disease 2019: a Juxtaposition of 2 pandemics. J Infect Dis. 2021;224(9):1455-1461. doi:10.1093/infdis/ jiab114

7. UN. Global humanitarian response plan COVID-19: United Nations Coordinated Appeal April-December 2020; 2020.

8. Asante Antwi H, Zhou L, Xu X, Mustafa T. Beyond COVID-19 pandemic: an integrative review of global health crisis influencing the evolution and practice of corporate social responsibility. In: Healthcare. Multidisciplinary Digital Publishing Institute; 2021.

9. World Health Organization. The World Health Report 2002: Reducing Risks, Promoting Healthy Life. World Health Organization; 2002. 
10. Pillay Y, Johnson L. World AIDS day 2020: reflections on global and South African progress and continuing challenges. South Afr J HIV Med. 2021;22(1):1-5. doi:10.4102/sajhivmed.v22i1.1205

11. USAID. Global health areas HIV and aids; 2021, Available from: https://www.usaid.gov/global-health/health-areas/hiv-and-aids. Accessed December 20, 2021.

12. Jewell BL, Mudimu E, Stover J, et al. Potential effects of disruption to HIV programmes in sub-Saharan Africa caused by COVID-19: results from multiple mathematical models. Lancet HIV. 2020;7(9): e629-e40. doi:10.1016/S2352-3018(20)30211-3

13. Santos G-M, Ackerman B, Rao A, et al. Economic, mental health, HIV prevention and HIV treatment impacts of COVID-19 and the COVID-19 response on a global sample of cisgender gay men and other men who have sex with men. AIDS Behav. 2021;25 (2):311-321. doi:10.1007/s10461-020-02969-0

14. Frontline AIDS. Coronavirus covid 19 impact on HIV. Available from: https://frontlineaids.org/were-on-the-frontline-of/coronaviruscovid-19-impact-hiv/-. Accessed December 20, 2021.

15. Ethiopian Federal Ministry of Health. National Comprehensive Covid19 Management Handbook. Ethiopian Federal Ministry of Health; 2020.

16. World Health Organization. COVID-19 strategy update. Geneve, Schweiz; 2020.

17. McMahon DE, Peters GA, Ivers LC, Freeman EE. Global resource shortages during COVID-19: bad news for low-income countries. PLoS Negl Trop Dis. 2020;14(7):e0008412. doi:10.1371/journal.pntd.0008412

18. World Health Organization. Maintaining Essential Health Services: Operational Guidance for the COVID-19 Context: Interim Guidance, 1 June 2020. World Health Organization; 2020.

19. Medina N, Alastruey-Izquierdo A, Bonilla O, et al. Impact of the COVID-19 pandemic on HIV care in Guatemala. Int $J$ Infect Dis. 2021;108:422-427. doi:10.1016/j.ijid.2021.06.011

20. Krakower D, Solleveld P, Levine K, Mayer K. Impact of COVID-19 on HIV preexposure prophylaxis care at a Boston community health center. J Int AIDS Soc. 2020;23:176-177.

21. Davey DLJ, Bekker L-G, Mashele N, Gorbach P, Coates TJ, Myer L. PrEP retention and prescriptions for pregnant women during COVID-19 lockdown in South Africa. Lancet HIV. 2020;7(11): e735. doi:10.1016/S2352-3018(20)30226-5
22. Gumbo O. COVID-19 lockdown measures on Zimbabwean populace. Adv Soc Sci Res J. 2020;7(7):797-814. doi:10.14738/assrj.77.8728

23. Mbulaje P, Nayupe SF, Munharo S, Nkwanju C, Banda C, LuceroPrisno III DE. Effects of COVID-19 on HIV services: the case of Malawi. PAMJ-One Health. 2021;5(1). doi:10.11604/pamj-oh. 2021.5.1.27607

24. Sandy C, Takarinda K, Timire C, et al. Preparing national tuberculosis control programmes for COVID-19. Int J Tuberc Lung Dis. 2020;24(6):634-636. doi:10.5588/ijtld.20.0200

25. Krubiner C, O’Donnell M, Kaufman J, Bourgault S. Addressing the COVID-19 crisis's indirect health impacts for women and girls. Center Glob Dev. 2021;4:1.

26. Cilliers J, Oosthuizen M, Kwasi S, et al. Exploring the impact of COVID-19 in Africa: a scenario analysis to 2030. ISS. 2020;1 (24): $1-40$.

27. Dorward J, Khubone T, Gate K, et al. The impact of the COVID-19 lockdown on HIV care in 65 South African primary care clinics: an interrupted time series analysis. Lancet HIV. 2021;8(3):e158-e65. doi:10.1016/S2352-3018(20)30359-3

28. Nyashanu M, Chireshe R, Mushawa F, Ekpenyong MS. Exploring the challenges of women taking antiretroviral treatment during the COVID-19 pandemic lockdown in peri-urban Harare, Zimbabwe. Int J Gynecol Obstet. 2021;154(2):220-226. doi:10.1002/ijgo.13771

29. Abraham SA, Berchie GO, Doe PF, Agyare E, Addo SA, Obiri-Yeboah D. Effects of COVID-19 pandemic on ART Service delivery: perspectives of healthcare workers in a Teaching Hospital in Ghana. BMC Health Serv Res. 2021;21(1):1-10. doi:10.1186/s12913-021-07330-2

30. Chilot D, Woldeamanuel Y, Manyazewal T. COVID-19 burden on HIV patients attending antiretroviral therapy in Addis Ababa, Ethiopia: a multicenter cross-sectional study. 2021.

31. Desta AA, Woldearegay TW, Gebremeskel E, et al. Impacts of COVID-19 on essential health services in Tigray, Northern Ethiopia: a pre-post study. PLoS One. 2021;16(8):e0256330. doi:10.1371/journal.pone.0256330
HIV/AIDS - Research and Palliative Care

\section{Publish your work in this journal}

HIV/AIDS - Research and Palliative Care is an international, peerreviewed open-access journal focusing on advances in research in HIV, its clinical progression and management options including antiviral treatment, palliative care and public healthcare policies to

\section{Dovepress}

control viral spread. The manuscript management system is completely online and includes a very quick and fair peer-review system, which is all easy to use. Visit http://www.dovepress.com/testimonials.php to read real quotes from published authors. 